\title{
Early Structure Formation and Reionization in a Cosmological Model with a Running Primordial Power Spectrum
}

\section{Citation}

Yoshida, Naoki, Aaron Sokasian, Lars Hernquist, and Volker Springel. 2003. “Early Structure Formation and Reionization in a Cosmological Model with a Running Primordial Power Spectrum." The Astrophysical Journal 598 (1): 73-85. https://doi.org/10.1086/378852.

\section{Permanent link}

http://nrs.harvard.edu/urn-3:HUL.InstRepos:41381764

\section{Terms of Use}

This article was downloaded from Harvard University's DASH repository, and is made available under the terms and conditions applicable to Other Posted Material, as set forth at http:// nrs.harvard.edu/urn-3:HUL.InstRepos:dash.current.terms-of-use\#LAA

\section{Share Your Story}

The Harvard community has made this article openly available. Please share how this access benefits you. Submit a story.

Accessibility 
TO APPEAR IN APJ

Preprint typeset using $\mathrm{LAT}_{\mathrm{E}} \mathrm{X}$ style emulateapj v. 04/03/99

\title{
EARLY STRUCTURE FORMATION AND REIONIZATION IN A COSMOLOGICAL MODEL WITH A RUNNING PRIMORDIAL POWER SPECTRUM
}

\author{
NAOKI Yoshida ${ }^{1,2}$, Aaron SoKasian ${ }^{1}$, LARs Hernquist ${ }^{1}$ \\ ${ }^{1}$ Harvard-Smithsonian Center for Astrophysics, 60 Garden Street, Cambridge MA02138 \\ ${ }^{2}$ National Astronomical Observatory Japan, Mitaka, Tokyo 181-8588, Japan \\ AND \\ VOLKER SPRINGEL ${ }^{3}$ \\ ${ }^{3}$ Max-Planck-Institut für Astrophysik, Karl-Schwarzschild-str. 1, D-85740 Garching bei München, Germany \\ To appear in ApJ
}

\begin{abstract}
We study high redshift structure formation and reionization in a $\Lambda$ CDM universe under the assumption that the spectral power index of primordial density fluctuations is a function of length scale. We adopt a particular formulation of the "running" spectral index (RSI) model as suggested by the combined analysis of the recent WMAP data and two other large-scale structure observations. We carry out high resolution cosmological simulations and use them to study the formation of primordial gas clouds where the first stars are likely to form.

While early structure forms hierarchically in the RSI model, quite similarly to the standard power-law $\Lambda \mathrm{CDM}$ model, the reduced power on small scales causes a considerable delay in the formation epoch of low mass $\left(\sim 10^{6} M_{\odot}\right)$ "mini-halos" compared to the $\Lambda$ CDM model. The abundance of primordial star-forming gas clouds in such halos also differs by more than an order of magnitude at $z>15$ between the two models. The extremely small number of gas clouds in the RSI model indicates that reionization is initiated later than $z<15$, generally resulting in a smaller total Thomson optical depth than in the $\Lambda \mathrm{CDM}$ model.

By carrying out radiative transfer calculations, we also study reionization by stellar populations formed in galaxies. We show that, in order to reionize the Universe by $z \sim 7$, the escape fraction of ultraviolet photons from galaxies in the RSI model must be as high as 0.6 throughout the redshift range $5<z<18$ for a stellar population similar to that of the local Universe. Even with a top-heavy intial mass function representing an early population of massive stars and/or an extraordinarily high photon emission rate from galaxies, the total optical depth can only be as large as $\tau_{e} \sim 0.1$ for reasonable models of early star-formation. The RSI model is thus in conflict with the large Thomson optical depth inferred by the WMAP satellite.
\end{abstract}

Subject headings: cosmology:theory - early Universe - reionization of the Universe

\section{INTRODUCTION}

The origin of matter density fluctuations in the Universe is one of the fundamental problems in cosmology. The so-called standard theory of structure formation posits that the present-day clumpy appearance of the Universe developed through gravitational amplification of an initially very smooth, but perturbed matter distribution. Recent observations provide a consistent picture of the largescale matter distribution in the early Universe; fluctuations arise from adiabatic perturbations whose statistics are described by a Gaussian field, as predicted by popular inflationary theories. The inflationary models also predict a scale-invariant power spectrum of primordial density fluctuations. In fact, the generic inflationary prediction is nearly but not precisely scale-invariant density fluctuations; i.e. the fluctuation power spectrum scales as $P(k) \propto k^{n}$ with $n \approx 1$ (see Lyth \& Riotto 1999 for a recent review). The tilt, or the exact value for $n$, has attracted considerable attention in theories of the physics of the early Universe (Covi \& Lyth 1999), in astronomical observations (Croft et al. 2002; Hannestad et al. 2002; Seljak, McDonald \& Makarov 2003) and in the context of structure formation (Zentner \& Bullock 2002).

The spectral index of the primordial power, $n$, may even be a function of length scale. This "running" of the spectral index modulates the relative amplitudes of density fluctuations on large to small scales. Interestingly, the combined analysis of the first-year Wilkinson Microwave Anisotropy Probe (WMAP) data, the $2 \mathrm{dF}$ galaxy redshift survey, and Lyman- $\alpha$ forest observations favors a cosmological model with a "running" of the primordial power spectrum (Spergel et al. 2003; Peiris et al. 2003). Although the WMAP data alone gives a best-fit power law $\Lambda$ CDM model with the spectral index $n \approx 1$ and the fluctuation amplitude $\sigma_{8}=0.9$, the Lyman- $\alpha$ forests observations (Croft et al. 1998, 2002; McDonald et al. 2000; Gnedin \& Hamilton 2002) consistently favor a lowamplitude model on scales $1 \lesssim k\left[\mathrm{Mpc}^{-1}\right] \lesssim 10$, which can be reconciled if the primordial power spectrum has a mild tilt $(n<1)$ or a negative running $(d n / d \ln k<0)$. For a given normalization on a large scale, models with negative running predict progressively reduced linear power on small scales (Kosowsky \& Turner 1995; Hannestad et al. 2002).

Suppressing linear power on small, galactic to sub- 
galactic scales may offer a resolution to the often-claimed problems of Cold Dark Matter (CDM) models (e.g. Moore et al. 1999). It could alleviate apparent discrepancies between the observed structure of dark halos and predictions from CDM models (Zentner \& Bullock 2003). On the other hand, too much reduction of small-scale power may result in a conflict with other observations. Dalal \& Kochanek (2002) and Chiba (2002) argue that the abundance of dark matter substructure in several galactic lens systems inferred from observed flux anomalies is consistent with predictions from the standard $\Lambda \mathrm{CDM}$ model (see however Bullock \& Zentner 2002 for an attempt to constrain some variant models). In models with reduced small scale power, nonlinear objects with characteristic mass $10^{6}-10^{9} M_{\odot}$ form late, and hence reionization due to photons from stellar sources formed in low mass systems is expected to occur late (Somerville, Bullock \& Livio 2003). To the contrary, the measurement of TE polarization by WMAP suggests a large Thomson optical depth $\tau_{e}=0.17 \pm 0.04$, implying that reionization took place as early as $z_{\text {reion }} \sim 17$ (Kogut et al. 2003).

Somerville et al. (2003) argue that models like the RSI one we consider here are inconsistent with the inferred early reionization epoch. Yoshida et al. (2003b) studied early structure formation in a warm dark matter model and showed that suppressing the linear power even on very small scales $\left(k>100 \mathrm{Mpc}^{-1}\right)$ can considerably affect the formation of high redshift star-forming gas clouds, making early reionization by massive, metal-free stars unlikely to occur early on. Chiu, Fan \& Ostriker (2003) argue that a large optical depth $\tau_{e}>0.1$ may require exotic radiation sources other than a normal stellar population. Avelino \& Liddle (2003) also conclude that running of the primordial power systematically delays the reionization epoch. Overall, these theoretical studies suggest that, if the large Thomson optical depth observed by the WMAP satellite is confirmed, the standard power-law $(n \approx 1) \Lambda \mathrm{CDM}$ model is favored over other models with reduced small scale power. Thus, the WMAP results appear to suggest a somewhat contradictory cosmological model. Since the major reason to favor power-law $\Lambda \mathrm{CDM}$ in this context is the inferred early epoch of reionization, it is clearly important to study and compare quantitatively details of the reionization process in the two cosmologies, the RSI model and a power law $\Lambda$ CDM universe.

In the present paper, we study early structure formation in RSI and $\Lambda$ CDM universes using cosmological $N$-body/hydrodynamic simulations. By combining these dynamical models with radiative transfer calculations, we compute the epoch of reionization by various stellar sources in the two cosmologies and compare the Thomson optical depth to that measured by the WMAP satellite.

In particular, at high redshifts, $z \gtrsim 15$, we consider the implications of an early population of massive, metal-free stars (the "first stars"), which have been hypothesized to form in "mini-halos" $\left(\sim 10^{6} M_{\odot}\right)$. We refer to the sites where these stars can form as "primordial gas clouds;" i.e. dense regions of cold gas which accumulate through the action of cooling by molecular hydrogen. In order to identify these primordial gas clouds, our simulations follow the non-equilibrium chemical reactions of a chemically pristine gas and molecular hydrogen cooling. In an earlier study
(Yoshida et al. 2003a), we found that non-equilibrium effects associated with dynamical heating play a critical role in regulating the supply of molecular gas, contrary to the assumptions commonly made in semi-analytic studies of this process. Hence, it is necessary to follow the dynamics of structure formation explicitly to reliably estimate the abundance of early stars. It is also important to specify the source location accurately when carrying out radiative transfer caculations. Unlike semi-analytic methods, direct hydrodynamic simulations enable us to locate plausible star-forming sites robustly in a cosmological volume. As we discuss later in the present paper, the distribution of the sources strongly affects the overall topology of the ionized regions.

We show that the reduced small scale power in the RSI model causes a considerable delay in the formation epoch of low mass halos and the primordial gas clouds within them compared to a $\Lambda$ CDM counterpart. In view of this result, it appears unlikely that massive stars in mini-halos can contribute significantly to reionization in cosmologies with a running spectral index, at least for those with parameters similar to what is inferred from the joint WMAPext + 2dFGRS + Lyman- $\alpha$ forest analysis (Spergel et al. 2003). To study the impact of ordinary ("Population II") stars formed in galaxies and protogalaxies, we carry out ray tracing calculations of reionization by stellar sources. For an initial mass function (IMF) similar to that in local galaxies, reionization can be completed at sufficiently early times to account for the Lyman- $\alpha$ optical depths measured in quasars at $z>6$, but in the case of the RSI model only if a large fraction of ionizing photons are able to escape from galaxies. Furthermore, because reionization occurs late in this context, the Thomson optical depth is too small to be compatible with that suggested by the WMAP measurements.

Finally, we consider the possibility that the production rate of ionizing photons could be boosted in galaxies and protogalaxies with a top-heavy IMF. In this case, Thomson optical depths approaching that favored by the WMAP analysis are possible, but only if the parameters describing these stars are pushed to extreme values.

The paper is organized as follows. We first describe the cosmological simulations we use to study the abundance of high-redshift star-forming systems in $\S 2$. We present the simulation results in $\S 3$. In $\S 4$, we discuss the possible effect of the finite size of our simulation volume. In $\S 5$, the abundance of primordial gas clouds and implications are described. There, we also give a brief description of the basic relation between reionization epoch and the total Thomson optical depth. In $\S 6$ we show the results of radiative transfer calculations in which we consider ordinary stellar populations in galaxies and protogalaxies as radiation sources. A summary and discussion are given in $\S 7$. 

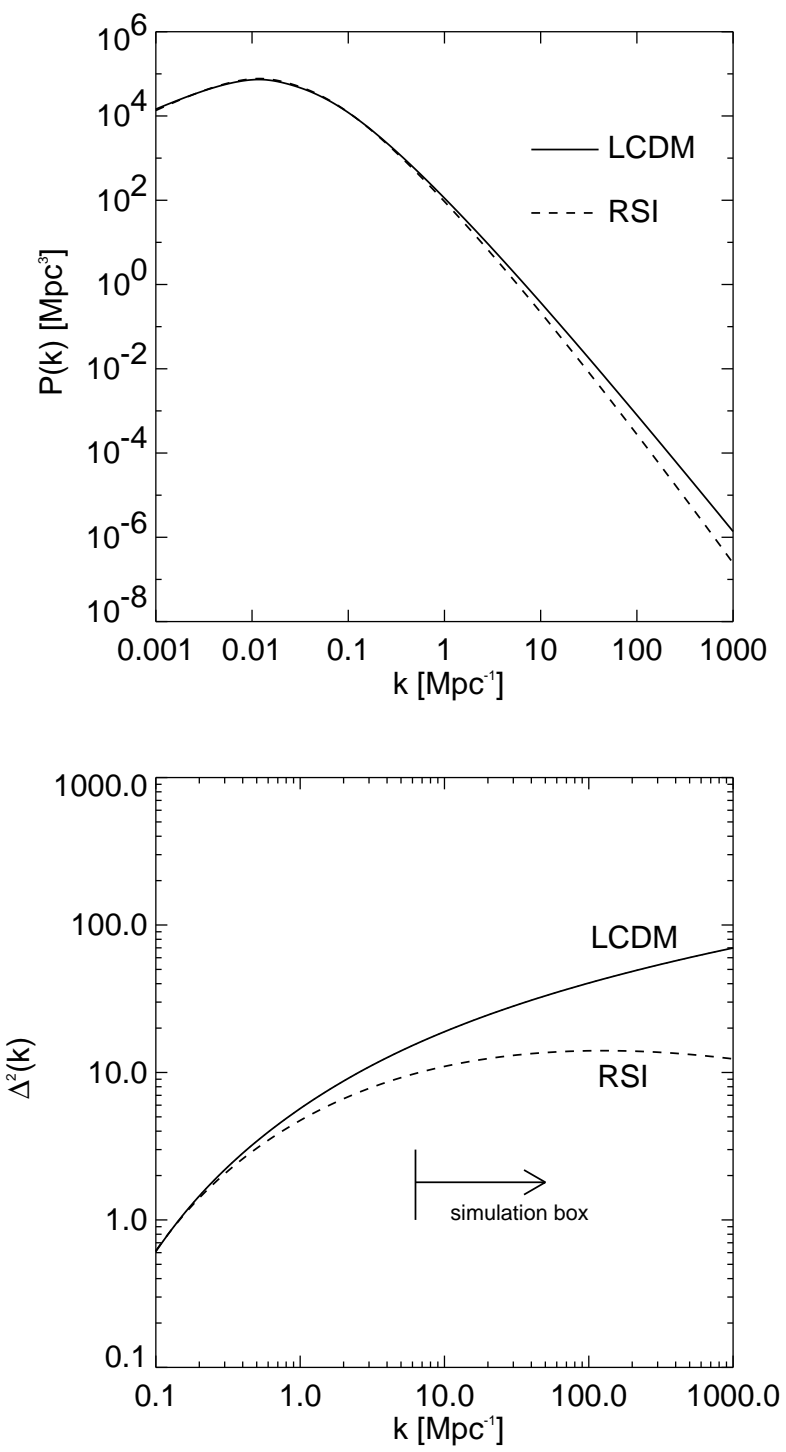

FIG. 1. - The linear power spectra for the power law $\Lambda \mathrm{CDM}$ model (solid line) and for the RSI model (dashed line). In the bottom panel we plot the variance $\Delta^{2}(k)$ linearly extrapolated to the present epoch for the two models. Note the difference in the plotted range in $k$ between the top and the bottom panels. The arrow in the bottom panel indicates the length scales our simulations probe.

\section{THE $N$-BODY/HYDRODYNAMIC SIMULATIONS}

We use the parallel Tree-PM solver GADGET2 combined with smoothed particle hydrodynamics (SPH) in our simulations. Our treatment of SPH employs the conservative entropy formulation of Springel \& Hernquist (2002), which offers several distinct advantages over previous versions of SPH. In particular, because the energy equation is written with the entropy as the independent thermodynamic variable, as opposed to the thermal energy, the ' $p \mathrm{~d} V$ ' term is not evaluated explicitly, reducing noise from smoothed estimates of e.g. the density. By including terms involving derivatives of the density with respect to the particle smoothing lengths, this approach explicitly conserves entropy (in regions without shocks), even when smoothing lengths evolve adaptively, avoiding the problems noted by e.g. Hernquist (1993). Furthermore, this formulation moderates the overcooling problem present in earlier formulations of SPH (Yoshida et al. 2002; see also Pearce et al. 1999, Croft et al. 2001).

The simulations follow the non-equilibrium reactions of nine chemical species $\left(\mathrm{e}^{-}, \mathrm{H}, \mathrm{H}^{+}, \mathrm{He}, \mathrm{He}^{+}, \mathrm{He}^{++}, \mathrm{H}_{2}\right.$, $\mathrm{H}_{2}^{+}, \mathrm{H}^{-}$) using the reaction coefficients compiled by Abel et al. (1997). We use the cooling rate of Galli \& Palla (1998) for molecular hydrogen cooling. Further simulation details are found in Yoshida et al. (2003a,b). The simulations employ $2 \times 324^{3}$ particles in a comoving volume of $1 \mathrm{Mpc}$ on a side. We work with $\Lambda$-dominated cosmologies with matter density $\Omega_{\mathrm{m}}=0.3$, cosmological constant $\Omega_{\Lambda}=0.7$ and the Hubble constant at the present time $h=0.7$ in units of $100 \mathrm{~km} \mathrm{~s}^{-1} \mathrm{Mpc}^{-1}$. We set the baryon density to $\Omega_{\mathrm{b}}=0.04$ and the normalization parameter to $\sigma_{8}=0.9$. The transfer functions are computed by the Boltzmann code of Sugiyama (1995) for the adopted cosmology and initial conditions are realized as described in Yoshida, Sugiyama \& Hernquist (2003). The primordial power spectrum for the $\Lambda \mathrm{CDM}$ case is assumed to be $P(k) \propto k$, whereas that of the RSI model is given by $P(k) \propto k^{n_{\mathrm{s}}}$ with

$$
n_{\mathrm{s}}=n_{\mathrm{s}}\left(k_{0}\right)+\frac{1}{2} \frac{\mathrm{d} n_{\mathrm{s}}}{\mathrm{d} \ln k} \ln \left(\frac{k}{k_{0}}\right)
$$

Specifically, we choose $k_{0}=0.05 \mathrm{Mpc}^{-1}, n_{\mathrm{s}}\left(k_{0}\right)=0.93$, and $d n_{s} / d \ln k=-0.03$, as indicated by the WMAPext $+2 \mathrm{dF}-$ GRS + Lyman- $\alpha$ analysis (Spergel et al. 2003). Note that we adopt the same normalization, $\sigma_{8}=0.9$, for both the power law $\Lambda$ CDM model and the Running Spectral Index model. Hereafter, we refer to the conventional power law $\Lambda \mathrm{CDM}$ model simply as the " $\Lambda \mathrm{CDM}$ model," and to the other as the "RSI model".

Figure 11 shows the linear power spectra $P(k)$ for the two models. In the bottom panel, we plot the variance per unit logarithmic interval in $k, \Delta^{2}(k)=k^{3} P(k) /\left(2 \pi^{2}\right)$, to better describe the difference between the two models in the range $0.1<k\left[\mathrm{Mpc}^{-1}\right]<1000$. Since the input power spectra are normalized at a length scale larger than the simulation boxsize, the amplitudes of the density fluctuations realized in the RSI simulation are smaller than in the $\Lambda \mathrm{CDM}$ simulation on all length scales. Hence, the density fluctuations on large scales $(k \sim 10)$ in the RSI model should evolve similarly to those in $\Lambda$ CDM but with a slight delay in time. We note that, while the boxsize of our simulations is sufficiently large to ensure that the fundamental fluctuation mode in the simulations remains in the linear regime at the early epochs we simulate, it may not sample a fair volume of the Universe. We will discuss finite boxsize effects in $\S 4$.

Figure 2 shows the projected gas distribution at $z=22$ (top panels) and at $z=17$ (bottom panels) for the two models. We choose these output redshifts such that the density fluctuation on the largest length scale in the RSI model at $z=17$ resembles that of the $\Lambda \mathrm{CDM}$ model at $z=22$. As expected, the RSI model appears as a delayed $\Lambda \mathrm{CDM}$ model on these scales. Also, rich small-scale structure develops early on only in the $\Lambda \mathrm{CDM}$ model, as can be inferred from the linear power spectrum (Figure 1). 

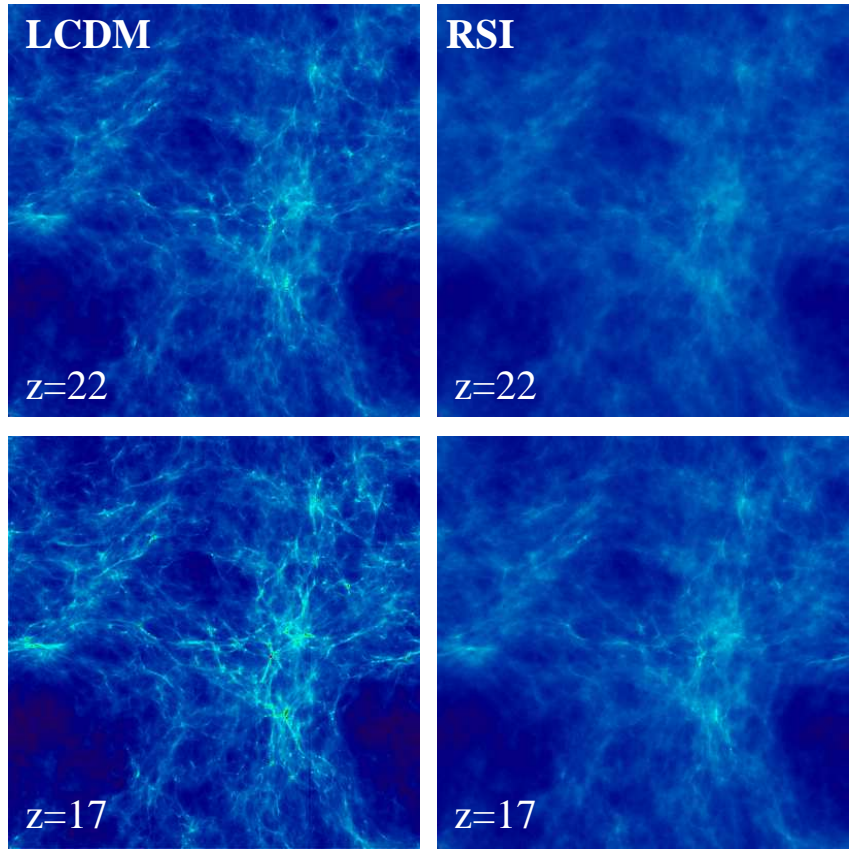

FIG. 2.- The projected gas density distribution at $z=22$ (top panels) and $z=17$ (bottom panels) for the $\Lambda$ CDM (left) and RSI (right) models.

\section{HALO ABUNDANCE}

Since the typical masses of the halos that host early primordial gas clouds are $5 \times 10^{5}-10^{7} M_{\odot}$ (Tegmark et al 1997; Machacek et al. 2001; Yoshida et al. 2003a), the abundance of such small mass halos is an important quantity to compare. In order to quantify the difference between the two models, we measure the mass function of dark matter halos. Whereas locating dark halos in pure $N$-body simulations is relatively simple using conventional techniques such as the friends-of-friends (FOF) algorithm, there are a few subtleties in identifying and assigning properties to halos in $N$-body/hydrodynamic simulations. Since our main objective is to compare the halo abundance between the two models, we avoid ambiguities by adopting a simple procedure, as follows.

We locate halos by running a FOF groupfinder with linking parameter $b=0.2$ on the dark matter particles in our simulations. We then assign the total mass to each group as the number of linked member particles multiplied by the scaled particle mass $m_{\text {part }}=m_{\mathrm{dm}}+m_{\text {gas }}$, where $m_{\mathrm{dm}}$ is the dark matter particle mass and $m_{\text {gas }}$ is the gas particle mass. We compute the Press-Schechter (PS) mass function to which we input the linear power spectrum for the dark matter component. (Note that, for our simulations, separate transfer functions are used for the dark matter and gas components in setting-up the initial conditions [for details, see Yoshida, Sugiyama \& Hernquist 2003].) We compare the mass function of the halos identified in this manner at $z=17$ and $z=22$ in Figure [3] . For both models, the measured mass function is overall well fitted by the PS mass function (the solid line for the $\Lambda \mathrm{CDM}$ model and the dashed line for the RSI model). Again, the RSI model at $z=17$ resembles the $\Lambda$ CDM model at $z=22$ with the abundance of low-mass halos being appreciably small.
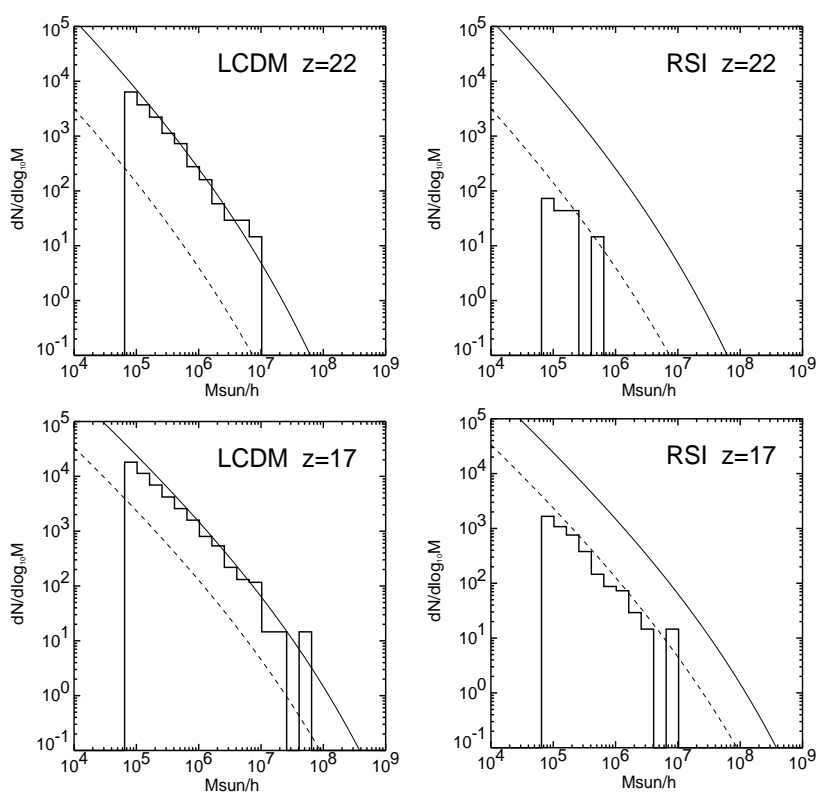

FIG. 3. - The mass function for the $\Lambda$ CDM model (left) and for the RSI model (right) at $z=17$ and $z=22$. The solid line is the Press-Schechter mass function for the $\Lambda \mathrm{CDM}$ model, and the dashed line is that for the RSI model.

The halo abundance in the RSI model appears to deviate slightly from the PS mass function. Although a similar feature is also seen in the $\Lambda \mathrm{CDM}$ model, the deviation from the PS mass function is more clearly noticeable for the RSI model. We discuss this issue together with finite boxsize effects in the next section.

\section{MASS VARIANCE}

It is well known that structure in CDM models grows hierarchically; i.e., smaller mass objects form earlier and merge to form bigger objects. While this can be easily inferred from the variance $\Delta^{2}(k)$ for the $\Lambda$ CDM model, which has larger amplitudes on smaller length scales (see Figure 1), the situation is less clear for the RSI model. As Figure 1 shows, the variance in the RSI model appears approximately constant over a range of scales $10<$ $k\left[\mathrm{Mpc}^{-1}\right]<1000$, indicating that nonlinear objects with widely different masses could form nearly at the same epoch. It is also worth mentioning that it is not clear whether the Press-Schechter theory can be used for such models. For the comparison shown in Figure 3. we calculated the PS mass function by formally inputting the linear power spectrum for the RSI model. Although the agreement shown in Figure 3 is reasonably good, it is not trivially expected. The flatness of the variance of the RSI model deserves particular attention also for another reason; the hierarchical nature of the formation of dark matter halos has a strong influence on the formation of primordial gas clouds (Yoshida et al. 2003a). Hence it is important to verify whether or not small mass $\left(\sim 10^{6} M_{\odot}\right)$ nonlinear objects in the RSI model grow hierarchically early on. For this purpose, it is more appropriate to work with a properly defined mass variance rather than the power spectrum.

\footnotetext{
*Only in Figure 3 and Figure 11 we use units that include the Hubble constant $h$, to be consistent with those commonly used; $h^{-1} M_{\odot}$ for mass and $\left(h^{-1} \mathrm{Mpc}\right)^{-3}$ for abundance.
} 


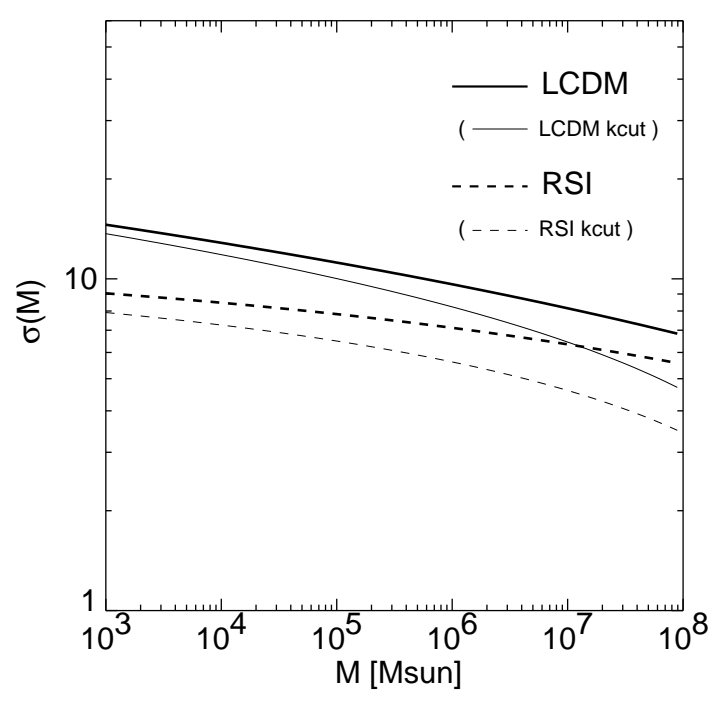

FIG. 4.- The mass variance computed from the power spectra linearly extrapolated to the present epoch. The thick solid line is for the $\Lambda$ CDM model, and the thick dashed line is for the RSI model. The thin lines show the effective mass variance as defined in equation (3) for each model.

We compute the mass variance as

$$
\sigma^{2}(M)=\frac{1}{2 \pi^{2}} \int P(k) W^{2}(k R) k^{2} \mathrm{~d} k,
$$

where the top-hat window function is given by $W(x)=$ $3\left(\sin (x) / x^{3}-\cos (x) / x^{2}\right)$. Figure 4 shows $\sigma(M)$ for the two models. In the plotted range, the mass variance in the RSI model monotonically increases toward smaller mass scales, indicating that less massive objects form at earlier epochs; i.e., structure formation is still expected to be "bottom-up". Note that the RSI model adopted in the present paper predicts an effective slope of the power spectrum smaller than -3 for $k \gg 200$. On very small length scales $(k>1000)$, or equivalently on low mass scales $\left(M<1000 M_{\odot}\right)$, structure formation should then proceed differently from the "bottom-up" picture. Owing to the flattening of the variance, the characteristic mass scale of the first non-linear objects may not be well defined. Halos with masses $10-1000 M_{\odot}$ should collapse first and larger objects will be formed through mergers of these small halos. Simulations with a substantially higher mass resolution are needed to reveal how halos with such very low masses form, although it will not be important for the formation of halos with masses $\sim 10^{6} M_{\odot}$ that are crucial for primordial gas cloud formation.

Let us now examine the consequences of the finite box size of our simulations. We quantify the effect by computing an effective variance

$$
\sigma_{\text {eff }}^{2}(M)=\frac{1}{2 \pi^{2}} \int_{k_{\min }} P(k) W^{2}(k R) k^{2} \mathrm{~d} k,
$$

where we set the lower boundary $k_{\min }$ to exclude the contribution from large-scale density fluctuations which are missing in our simulations. We simply set $k_{\min }=2 \pi / L_{\text {box }}$ with $L_{\text {box }}$ being the simulation box sidelength. The thin lines in Figure 4 are the effective mass variance computed in this manner. For both the models, $\sigma_{\text {eff }}(M)$ is

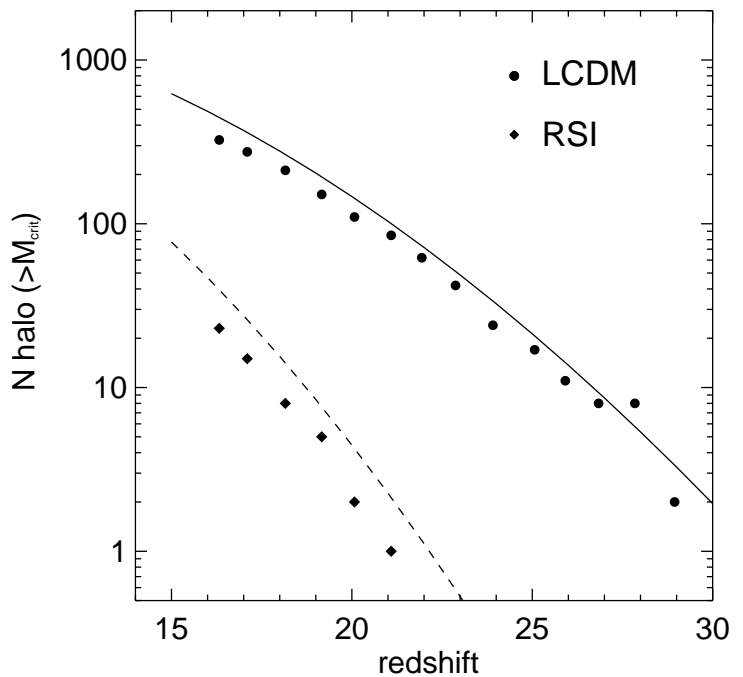

FIG. 5. - The number of halos with mass greater than $7 \times 10^{5} M_{\odot}$ per cubic mega-parsec (comoving) volume. The solid line is the analytic estimate obtained from the Press-Schechter mass function for the $\Lambda$ CDM model, and the dashed line is for the RSI model.

slightly smaller than $\sigma(M)$ on the relevant mass scales $\left(\sim 10^{5}-10^{6} M_{\odot}\right)$, and thus a systematic delay in the characteristic formation epoch (or a slightly lower abundance at a given epoch) is expected. This may account for the small discrepancy in the mass function plotted in Figure 3. Since we use linearly extrapolated power spectra to compute $\sigma(M)$ and $\sigma_{\text {eff }}(M)$, the precise effect of a finite boxsize remains still uncertain. The apparent difference shown in Figure 4 should be interpreted as qualitatively representing the finite boxsize effect. Note that the RSI model is more affected by the finite boxsize because the relative fractional power on large scales is bigger than in the $\Lambda$ CDM model (see Figure1). Systematic studies using larger box simulations are clearly needed to address this issue further.

By constructing a halo merger history using 30 simulation outputs dumped frequently between $17<z<25$, we also verified that almost all the halos that have masses greater than $7 \times 10^{5} M_{\odot}$ at $z=17$ grew monotonically in mass by repeated mergers and continuous accretion of smaller mass elements, quite similarly to what is found in the $\Lambda \mathrm{CDM}$ model at somewhat higher redshifts. As long as we are concerned with the abundance of low-mass halos, the results of our small box simulations appear to be in reasonable agreement with the Press-Schechter mass function. In Figure 5 we show the number of halos with masses greater than $M_{\text {crit }}=7 \times 10^{5} M_{\odot}$. We chose the threshold mass $M_{\text {crit }}$ based on the results of Yoshida et al. (2003a) for star-forming systems. Although the incomplete sampling of the halo mass function owing to the finite boxsize is appreciable at high redshift, $z>27$ for the $\Lambda$ CDM model and $z>20$ for the RSI model, overall the halo abundance is in agreement with the PS prediction. Thus, we conclude that the statistics we discuss in the present paper, such as the abundance of primordial gas clouds, are not significantly affected by the finite simulation boxsize. The apparent discrepancy in Figure 5 may be partly due to some inaccuracies of the analytic mass function itself (see Jenkins et al. 2001; Reed et al. 2003), 
although the simulation results of Jang-Condell \& Hernquist (2001) indicate that the PS mass function is a good approximation on these mass scales. An important point to note is that both the analytic estimate and the simulation results suggest that the number of of halos with mass $\sim 10^{6} M_{\odot}$, which are crucial for the primordial gas cloud formation, is an order of magnitude smaller in the RSI model.

Finally, we mention that a promising way of compensating for the missing large-scale power would be to employ the mode-adding procedure developed by Tormen \& Bertschinger (1996) and by Cole (1997). The method can effectively extend the dynamic range of cosmological simulations, and thus will be useful for studies of early structure formation in a cosmological context.

\section{CONTRIBUTION OF THE "FIRST STARS" TO REIONIZATION}

Numerical simulations (e.g. Abel, Bryan \& Norman 2002; Bromm, Coppi \& Larson 2002) suggest that the first stars in the Universe were unusually massive and formed in "mini-halos" at redshifts $z>20$, as a consequence of cooling by molecular hydrogen. It is customary to refer to early, massive stars as Population III. In what follows, we will distinguish between massive stars which may have formed in mini-halos by molecular cooling (the "first stars") from those which could have originated in larger halos where gas can cool by atomic processes. While the nature of the "first stars" has been studied in some detail, the stellar populations expected in slightly more massive halos (the "first galaxies") is unclear. With modest uncertainty, we can quantify the importance of the former to reionization, but numerous assumptions are required to account for "non-standard" IMFs in early protogalaxies, as we discuss later in the paper.

\subsection{Primordial gas cloud formation}

Owing to the delayed formation of "mini-halos" in the RSI model, the formation of primordial gas clouds is also expected to occur late. It is in these regions that significant quantities of molecular gas can accumulate, leading to the formation of massive, metal-free stars of the type modeled by e.g. Abel, Bryan \& Norman (2002) and Bromm, Coppi \& Larson (2002). We emphasize that nonequilibrium processes not typically accounted for in semianalytic estimates, like the dynamical heating effect identified by Yoshida et al. (2003a), significantly influence the abundance of these star-forming regions.

In Figure 6 we plot the number of gas clouds identified in the two simulations. We define groups of cold $(T<500 \mathrm{~K})$, dense $\left(n_{\mathrm{H}}>500 \mathrm{~cm}^{-3}\right)$ gas particles as "gas clouds". We locate the gas clouds by running a FOF groupfinder to the simulation gas particles with a small linking parameter $b=0.05$. We then discard from the groups gas particles that do not satisfy the above conditions. Figure [ 6 clearly shows that the total number of gas clouds in the simulated volume differs by more than an order of magnitude between the two models in the redshift range plotted. At $z=17$, we identified 66 gas clouds in the CDM model, whereas there is only one gas cloud found in the RSI model. We continued the RSI run down to $z=15$ to see if there is a rapid increase in the number of gas clouds. Although the increase in the number of gas clouds at $z \sim 14-17$ appears large, the total number is still much smaller than that of the $\Lambda \mathrm{CDM}$ model. As we argued in section 4, some halos grow very rapidly in the RSI model, which causes considerable perturbation to the formation and the growth of the primordial gas clouds within them. This may partly explain the extremely small number of gas clouds at $z \sim 15$ in the RSI model. It is interesting that the number of the gas clouds in the RSI model is even smaller than, or at most comparable to, the warm dark matter case we studied in Yoshida et al. (2003b). For the specific model

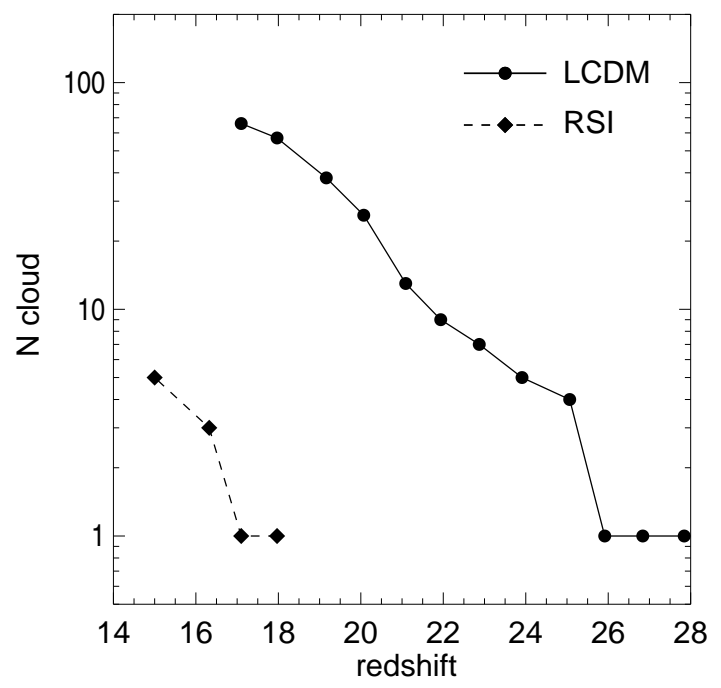

FIG. 6.- The number of primordial "gas clouds" where massive, metal free stars can form by molecular hydrogen cooling.

employed in Yoshida et al. (2003b) that assumes a single massive $\left(300 M_{\odot}\right)$, metal-free star is formed per gas cloud, reionization by the "first stars" is not completed at $z>15$ in the RSI model. In fact, with the very small number of sources, the ionized volume fraction rises only up to a few percent by $z=15$. This is clearly in disagreement with the WMAP result of $z_{\text {reion }} \sim 17$. Since the estimated uncertainty in the WMAP result of the reionization epoch is rather large (Kogut et al. 2003), it still remains to be seen whether the particular RSI model we consider is compatible with the WMAP data. Nevertheless, it is clear that the running of the power spectrum is not favored in terms of early reionization.

\subsection{Reionization epoch and the Thomson optical depth}

The differential optical depth to Thomson scattering in a small redshift interval $\mathrm{d} z$ is

$$
\mathrm{d} \tau_{e}=\sigma_{\mathrm{T}} n_{e}(z) c \frac{\mathrm{d} t}{\mathrm{~d} z} \mathrm{~d} z,
$$

where $\sigma_{\mathrm{T}}=6.65 \times 10^{-25} \mathrm{~cm}^{2}$ is the Thomson scattering cross-section, $n_{e}(z)$ is the mean electron number density at $z$, and $c$ is the speed of light. The mean electron number density is given by

$$
n_{e}(z)=n_{e}(0)(1+z)^{3} x_{e}(z),
$$

where $x_{e}$ is the ionization fraction. For a flat $\Lambda \mathrm{CDM}$ model, $H(z)=H_{0}\left[\Omega_{\Lambda}+\Omega_{\mathrm{m}}(1+z)^{3}\right]^{1 / 2}$. 


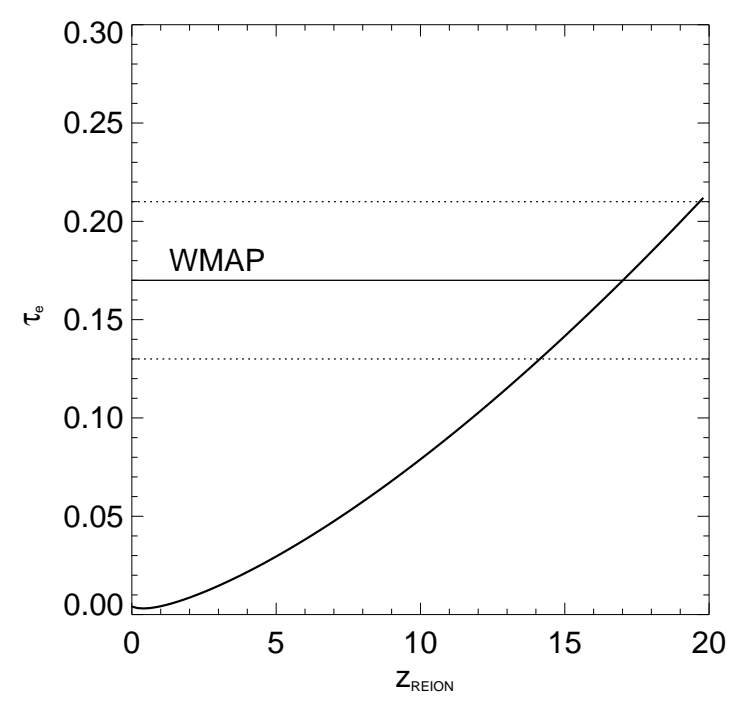

FIG. 7.- The optical depth to Thomson scattering to $z_{\text {reion }}$ when abrupt and complete reionization at $z_{\text {reion }}$ is assumed.

Putting $x_{e}=1$ for an interval between $z_{1}$ and $z_{2}$, we obtain the contribution as

$$
\left.\mathrm{d} \tau_{e}\right|_{z_{1} \rightarrow z_{2}}=\left.\frac{2}{3} \frac{\sigma_{\mathrm{T}} n_{e}(0) c}{\left(\Omega_{\mathrm{m}} H_{0}^{2}\right)^{1 / 2}}\left[(1+z)^{3}+\frac{\Omega_{\Lambda}}{\Omega_{\mathrm{m}}}\right]^{1 / 2}\right|_{z_{1}} ^{z_{2}} .
$$

Consider a simple case where abrupt and complete reionization occurs at $z$. Using the WMAP results for the cosmological parameters, $\Omega_{\mathrm{b}} h^{2}=0.0224$ and $\Omega_{\mathrm{m}} h^{2}=0.135$, and assuming a plasma of primordial abundance with, for example, fully ionized hydrogen and singly ionized helium, we obtain the total optical depth to redshift $z$ :

$$
\int_{0}^{z} \mathrm{~d} \tau_{e} \approx 0.0023\left[\left([1+z]^{3}+2.7\right)^{1 / 2}-1.93\right] .
$$

Figure 7 shows the total optical depth against reionization epoch at $z=z_{\text {reion }}$. The WMAP result is indicated at $\tau_{e}=0.17$ (solid line) with a $1-\sigma$ range $0.17 \pm 0.04$ (dotted line). The actual reionization history could be more complicated, but Figure 7 provides the useful insight that a large Thomson optical depth, say $\tau_{e}>0.13$, can be achieved only if reionization takes place at an early epoch, $z_{\text {reion }} \gtrsim 14$.

Our simulation of early structure formation in the RSI model showed that there are only 5 gas clouds at $z=15$ in the simulated volume. As Yoshida et al. (2003b) demonstrate, approximately 100 very massive stars must be turned on within $\sim 1$ recombination time in a $(1 \mathrm{Mpc})^{3}$ volume to cause complete reionization. Owing to the strong radiative feedback from the first stars, the number of stars formed in the primordial gas clouds is likely to be limited to one (Omukai \& Nishi 1999; Oh et al. 2001). Hence the number of "first stars" in the RSI model is far too small at $z>15$ to reionize the Universe. The epoch of complete reionization is certainly much later than $z \sim 15$, implying a small total optical depth $\tau_{e} \ll 0.13$.

In summary, primordial gas cloud formation which relies on molecular hydrogen cooling in low-mass, "mini-halos" appears to be extremely inefficient at $z>15$ in the RSI model and thus other sources, such as early protogalaxies, must contribute to reionization for this model to be compatible with the WMAP results. Furthermore, it is important to establish whether or not ordinary stellar sources in galaxies in the RSI model can account for reionization early enough to even be consistent with measurements of the Lyman- $\alpha$ optical depth in quasars at $z>6$.

\section{REIONIZATION BY STARS IN GALAXIES}

In the previous section, we have shown that low-mass mini-halos and primordial gas clouds which can harbor massive, metal free stars (the "first stars") do not form in sufficient numbers at high redshift in the RSI model to account for reionization at $z \gtrsim 15$. As we emphasize in $\S 5.2$ and Figure 7, reionization must occur early if the WMAP measurement of the Thomson optical depth is accurate.

The number of larger mass $\left(\sim 10^{7}-10^{9} M_{\odot}\right)$ halos is also predicted to be small for the RSI model. Thus, reionization by stars in systems that are the likely progenitors of galaxies will be less efficient than for a $\Lambda \mathrm{CDM}$ model, for similar assumptions about the stellar populations. In this section, we study reionization by stars in galaxies and proto-galaxies with total masses larger than $\sim 4 \times 10^{7} M_{\odot}$, where the gas can cool rapidly by atomic line transitions. The nature of the stars forming early in these environments is uncertain. It is possible, for example, that the IMF will be top-heavy, at least initially, perhaps increasing the production rate of ionizing photons per unit stellar mass compared to a conventional IMF inferred for stellar populations in the local Universe.

In what follows, we begin by considering star formation in halos in the RSI model by assuming that the stellar population is similar to that locally and is unevolving. Namely, we adopt a Salpeter (1955) IMF and normalize the star formation rate (SFR) so that it reproduces the Kennicutt (1998) law. For simplicity, we refer to this description as a "Population II" SFR. In $\S 6.4$ we examine the overall effect of relaxing these assumptions. However, it is already clear that the RSI model will have difficulty accounting for the WMAP estimate of the Thomson for any reasonable assumptions about star formation simply because structure forms too late in this scenario to produce a large $\tau_{e}$, as suggested by Figure 7 .

To study how reionization proceeds in the RSI model, we run multi-source radiative transfer simulations using the technique of Sokasian et al. (2001, 2003a,b). In order to locate radiation sources and to compute the IGM density field, we utilize existing outputs of a large volume $\Lambda \mathrm{CDM}$ simulation from Springel \& Hernquist (2003b), normalizing the star formation rate expected for the RSI model using the analytic description of Hernquist \& Springel (2003), which can be applied to any cosmology.

\subsection{The star-formation rate in the RSI model}

First, we compute the "generic" star-formation rate for the RSI model using the analytic model of Hernquist \& Springel (2003). Their approach describes the formation of dark matter halos using an analytic halo mass function (Sheth \& Tormen 1999; 2002), and employs simplified prescriptions for the cooling of gas and star-formation which are commonly used in semi-analytic models of galaxy formation (Kauffmann et al. 1999; Springel et al. 2001; Yoshida et al. 2002). This analytic formalism has been tested in detail by comparing its predictions to those from 


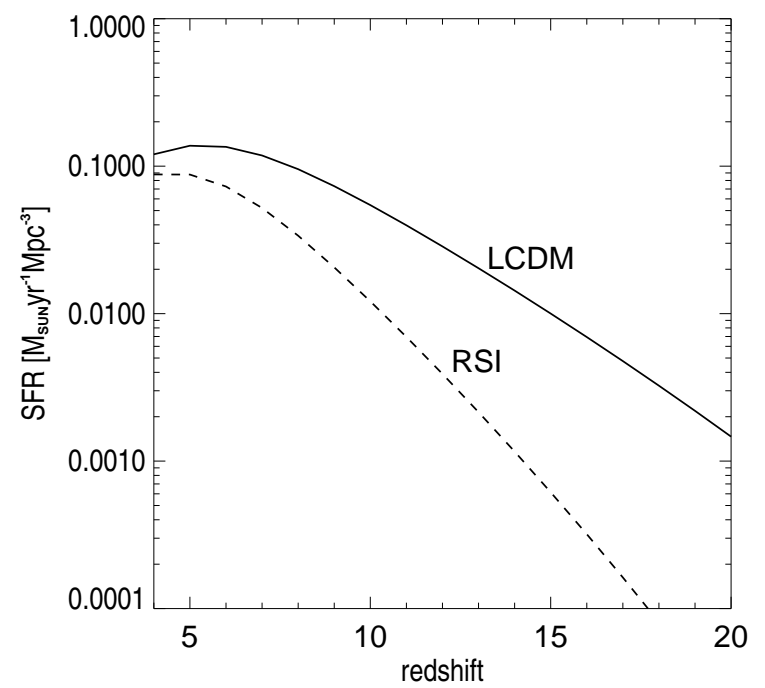

FIG. 8.- The star-formation rate density in galaxies ("Population II") as a function of redshift for the $\Lambda$ CDM (solid line) and RSI (dashed line) models.

cosmological simulations that include hydrodynamics and star formation (Springel \& Hernquist 2003b). A thorough description of the model is given by Hernquist \& Springel (2003) and references therein.

In particular, Hernquist \& Springel (2003) show that under rather general circumstances the star-formation rate density is well-approximated by the simple form

$$
\dot{\rho}_{*}(z)=\dot{\rho}_{*}(0) \frac{\chi^{p}}{1+\alpha(\chi-1)^{q} \exp \left(\beta \chi^{r}\right)},
$$

where

$$
\chi(z) \equiv\left(\frac{H(z)}{H_{0}}\right)^{2 / 3} .
$$

In equation (8), the factors $\beta$ and $r$ are fixed by the underlying cosmological power spectrum, the choices for $p$ and $q$ reflect the interplay between radiative cooling in halos and the expansion of the Universe, and the normalizing constants $\dot{\rho}_{*}(0)$ and $\alpha$ depend on the rate at which cold, dense gas is converted into stars.

The form of equation (8) is motivated by two competing effects which regulate star formation. At sufficiently high redshifts, collapsed gas is dense and cooling times are short so that star formation is only limited by the gravitational growth of massive halos. At low redshifts, the cooling time in halos increases owing to the decline in the mean density of the Universe by cosmic expansion. In this regime, the supply of star-forming gas is throttled by the expansion of the Universe.

Based on the multiphase description of star-forming gas described by Springel \& Hernquist (2003a), Hernquist \& Springel (2003) show that the appropriate values for the parameters in equation (8) for the $\Lambda \mathrm{CDM}$ model employed here are $p=2, q=3, r=7 / 4, \alpha=0.012, \beta=0.041$, and $\dot{\rho}_{*}(0)=0.013 M_{\odot} / \mathrm{yr} / \mathrm{Mpc}^{3}$.

For the RSI cosmology, we use the RSI linear power spectrum as input and follow the procedure described in Hernquist \& Springel (2003). We find that the resulting SFR density is again well-fitted by equation (8) for the choices $p=1.5, q=3.05, r=1.85, \alpha=0.01, \beta=0.057$, and $\dot{\rho}_{*}(0)=0.0176 M_{\odot} / \mathrm{yr} / \mathrm{Mpc}^{3}$.

In Figure 8 we compare the SFR density estimated in this manner for the two cosmologies. The RSI model predicts a significantly smaller SFR than the $\Lambda$ CDM model, in qualitative agreement with the semi-analytic estimates of Somerville et al. (2003). We use the forms for the SFR density in our ray-tracing calculatiosn only within the restricted redshift range $5<z<20$ to compute radiation source luminosities.

\subsection{Source luminosities}

The second step is to prescribe the luminosity of the sources ("galaxies") located in the simulations. Specifically, we use the outputs from Run Q5 of Springel \& Hernquist (2003b), which simulates a cube of $14.3 \mathrm{Mpc}$ on a side. We first calibrate the SFRs of individual sources identified in the simulation, and then convert these into luminosities. As in Sokasian et al. (2003a), we use the derived SFR (equation 8) as a reference to match the total SFR of the sources. We carry out the calibration procedure after selecting sources in the simulation outputs in the following two ways.

\subsubsection{Dim source model}

In this approach, we identify all the halos found in each output as "galaxies" and assign luminosities to them based on the actual star-formation rates which are computed in a dynamically consistent way in the cosmological simulation. The minimum mass of the sources is set by the resolution of the simulation, which is $M_{\min }=4.3 \times 10^{7} h^{-1} M_{\odot}$ in our case. The SFRs of the sources with masses close to the resolution limit are corrected in the same manner as in Sokasian et al. (2003a, §4.2.1). We then scale the SFRs of all the galaxies by multiplying by a constant factor $C_{\mathrm{L}}(z)$ such that the total SFR within the simulated volume matches that for the RSI model obtained from equation (8). Note that the scaling factor $C_{\mathrm{L}}(z)$ is kept constant for all the galaxies within one simulation output, but it is time-dependent.

\subsubsection{Massive halo model}

Since the halo abundance itself is lower in the RSI model than in the $\Lambda \mathrm{CDM}$ cosmology, it may be more appropriate to populate a smaller number of objects as radiation sources in the RSI model. Motivated by this, we first compute the number density of halos with virial temperature greater than $10^{4} \mathrm{~K}$ in which the gas can cool by atomic line transitions. The threshold mass is given by

$$
M_{\min }=10^{8}\left(\frac{1+z}{11}\right)^{-3 / 2} M_{\odot} .
$$

Then we use the halo mass function to compute the expected abundance of halos with mass greater than $M_{\min }$. Let us denote this number by $N_{\text {source }}(z)$. In the simulation output produced at $z$, we select $N_{\text {source }}(z)$ halos in order of their mass. Luminosities are assigned to the selected sources in proportion to their mass, $L \propto M_{\text {halo }}$ such that the total SFR summed over the selected sources matches the global SFR given by equation (8), with the parameter values appropriate for the RSI model. 


\subsubsection{Escape fraction}

Finally, the ionizing flux from the sources is modulated by the "escape fraction," $f_{\text {esc }}$, which describes the probability that individual photons can escape from the galaxy in which they were produced into the surrounding intergalactic medium (IGM). Sokasian et al. (2003a) studied reionization by stellar sources in the $\Lambda$ CDM universe by adopting several values for $f_{\text {esc }}$. Based on a detailed comparison with the observational results of the $z=6.28$ quasar (SDSS 1030.10+0524) in Becker et al. (2001), they conclude that $f_{\mathrm{esc}}=0.2$ is most consistent with the observed Lyman- $\alpha$ flux transmittance. We take this $f_{\text {esc }}=0.2$ model in Sokasian et al. (2003a) as our fiducial case for the $\Lambda \mathrm{CDM}$ model. For the RSI model, our zeroth order requirement is that reionization should be completed by $z=7$. After several experiments, we found that setting $f_{\text {esc }}>0.6$ is necessary for the RSI model to satisfy this condition. We thus set $f_{\text {esc }}=0.6$ as our basic choice for the RSI model and compare the results with the fiducial $\Lambda$ CDM model.

\subsection{Numerical results for "Population II" star formation}

We compute the total volume filling factor of the ionized medium $Q_{\mathrm{HII}}$ from the results of our ray-tracing calculations. Figure 9 shows the resulting ionized volume fraction, and Figure 10 shows the Thomson optical depth $\tau_{e}$ as a function of redshift. In the figures, we show only the results for our "massive-halo model" of source selection for the RSI model. Comparing the results for the dimsource model and the massive-halo model, we found that there is no substantial difference between the two in either $\tau_{e}(z)$ or $Q_{\mathrm{HII}}(z)$. We note, however, that the overall morphology of the ionized regions looks somewhat different between the two cases, because there are more sources in the dim-source model that reside in less dense regions. Nevertheless, for global quantities such as the total optical depth, the two models are found to be quite similar.

On the other hand, the results for the $\Lambda \mathrm{CDM}$ and the RSI models differ substantially. As Figure 9 shows, the ionized volume fraction in the RSI model remains small until $z \sim 9$, and then rapidly increases close to unity at $z \sim 7$. At later epochs, $z \lesssim 9$, the abundance of high mass $\left(\sim 10^{9} M_{\odot}\right)$ halos rapidly increases, and the total star-formation (and hence the photon production) is dominated by such large halos. This can also be understood in terms of the halo mass function at $z=7$ shown in Figure 11] By this time, the mass functions for the two models are already quite similar, with the difference being only a constant factor of roughly two (compare with the much larger difference at $z=17$ at smaller mass scales shown in Figure 3). Namely, the RSI model quickly catches up with the $\Lambda \mathrm{CDM}$ model in the abundance of halos around $z \sim 7-11$ (see also the increasing SFR in Figure 8), which causes the rapid increase in the ionized volume fraction as seen in Figure 9 Reionization is nearly completed $\left(Q_{\mathrm{HII}}>0.95\right)$ at $z=8$ in the $\Lambda$ CDM model, whereas it occurs at $z \approx 7.3$ in the RSI model. However, as shown in Figure 10 the resulting total optical depth $\tau_{e}=0.055$ for the RSI model is still small, owing to the small $Q_{\mathrm{HII}}$ at earlier epochs. We emphasize that a large Thomson optical depth can be achieved only if reionization occurs early $(\S 6)$. In other words, after reionization is completed, the additional

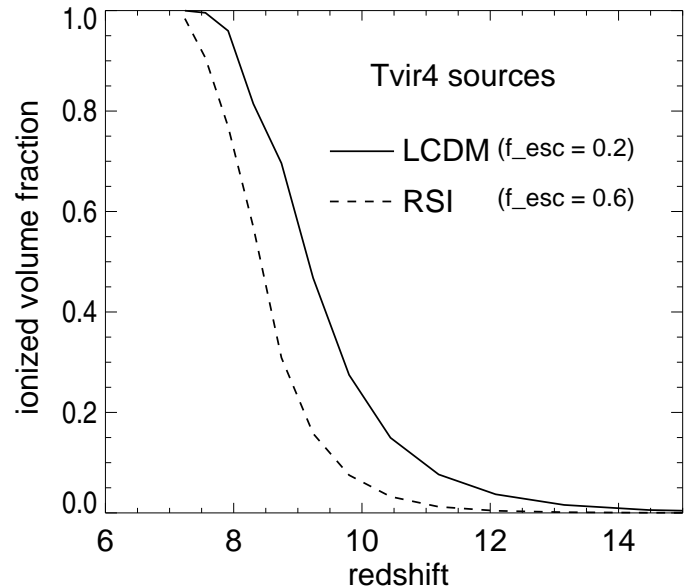

FIG. 9. - The ionized volume fraction for the $\Lambda$ CDM model with $f_{\text {esc }}=0.2$ (solid line) and for the RSI model with $f_{\text {esc }}=0.6$ (dashed line), for "Population II" stars.

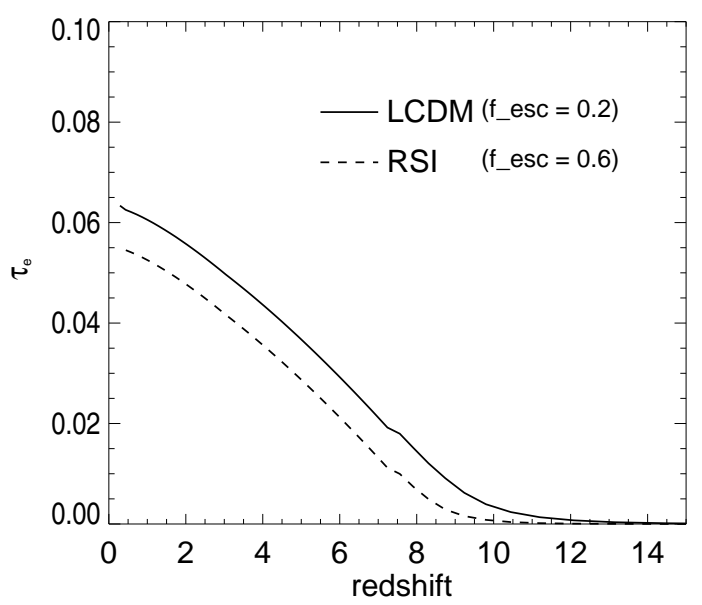

FIG. 10.- The Thomson optical depth computed from the outputs of our simulations for the $\Lambda \mathrm{CDM}$ model with $f_{\text {esc }}=0.2$ (solid line) and for the RSI model with $f_{\text {esc }}=0.6$ (dashed line) for "Population II" stars.

photon supply does not matter in the total optical depth as long as the IGM is kept almost fully ionized thereafter, as implied by Figure 7. (Note that here we discuss only hydrogen reionization.)

Our fiducial $\Lambda \mathrm{CDM}$ case with $f_{\text {esc }}=0.2$ predicts a larger total optical depth than the RSI model with $f_{\text {esc }}=0.6$ by $\Delta \tau_{e} \sim 0.01$. The difference mostly comes from the fact that $Q_{\mathrm{HII}}$ is still quite small at $z>9$ in the RSI model. Interestingly, none of the "conventional" scenarios we have considered for "Population II" star formation reproduces the claimed high optical depth from WMAP of $0.13<\tau_{e}<0.21(1-\sigma ;$ Kogut et al. 2003). It is clear that these models are inconsistent with the WMAP measurement and thus may need, for example, additional radiation sources to ionize the IGM early on.

In related work, we have shown that an early generation of massive, metal-free stars in mini-halos supplemented by "ordinary" star formation at lower redshifts to account for the spectra of high redshift quasars can yield a Thomson optical depth approaching that of the WMAP measurement in a $\Lambda$ CDM cosmology (Yoshida et al. 2003b, 


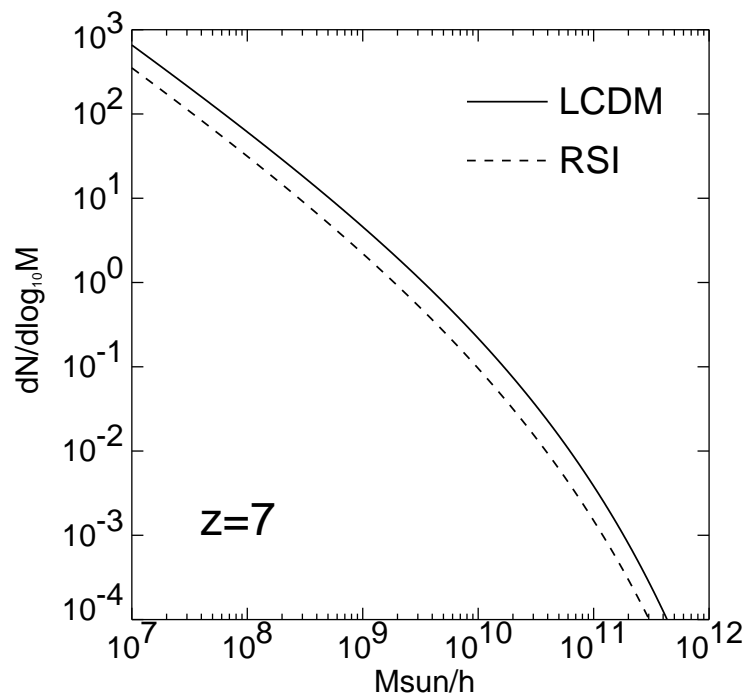

FIG. 11. - The Press-Schechter mass function at $z=7$.

Sokasian et al. 2003b), along the lines suggested by Haiman \& Holder (2003) and Cen (2003). However, it does not appear that a similar conclusion will obtain for the RSI model because of the low abundance of mini-halos at high redshifts, as we found in the case of a warm dark matter model with particle mass $10 \mathrm{keV}$ (Yoshida et al. 2003b).

\subsection{Star formation in galaxies with boosted photon emission}

The results described above indicate that the RSI model is incompatible with a high Thomson optical depth like that suggested by the WMAP measurements. A remaining loophole in this argument is that the stellar population in galaxies at early times may have differed from that in our underlying model so that a larger number of ionizing photons were produced from galaxies at $z>10$ than we assumed above (e.g. Ciardi, Ferrara \& White 2003; Wyithe \& Loeb 2003; Sokasian et al. 2003a). We first discuss such possibilities quantitatively, and then show numerical results for a model that enhances the photon production rate in galaxies.

For a Salpeter-type power-law IMF, the hydrogen ionizing photon emission rate of zero-metallicity stars is larger than that of a Population II (i.e. having metals) counterpart only by about 50\% (Thumlinson \& Shull 2000; see Leitherer et al. 1999 for low-metallicity cases.). The stellar IMF in the first galaxies may have had even an extreme top-heavy shape, with massive stars exclusively forming out of a primeval gas (Schwarzschild \& Spitzer 1953; Matsuda, Sato \& Takeda 1969; Yoshii \& Saio 1986; Larson 1998; Abel, Bryan \& Norman 2002; Bromm, Coppi \& Larson 2002). Very massive Population III stars are efficient emitters of UV photons, with the hydrogen ionizing photon emission rate per stellar mass being up to 10-20 times higher than for ordinary stellar populations (Bromm, $\mathrm{Ku}-$ dritzki \& Loeb 2001; Schaerer 2002). It is thus conceivable that, in early galaxies, the photon emission rate per stellar mass was $\sim 10$ times higher than that of the "Population II" case we considered above.

More specifically, the predicted star-formation rate for the RSI model shown in Figure 8 is

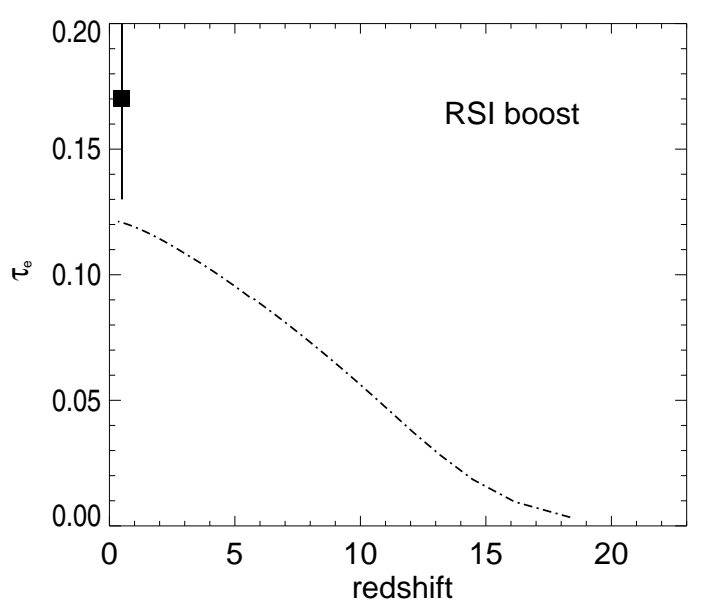

FIG. 12.- The Thomson optical depth for the RSI model in which the photon emission rate is "boosted" according to equation (11). The filled square is the WMAP result with its $1-\sigma$ error bar.

$\sim 5 \times 10^{-4} M_{\odot} \mathrm{yr}^{-1} \mathrm{Mpc}^{-3}$ at $z=15$. Assuming a constant conversion factor of $10^{53} \mathrm{~s}^{-1}$ ionizing photons per star-formation rate of $1 M_{\odot} \mathrm{yr}^{-1}$ for an ordinary stellar population with solar metallicity (Madau, Haardt \& Rees 1999), we obtain a total rate of $\sim 5 \times 10^{49}$ ionizing photons per second per cubic comoving $1 \mathrm{Mpc}$ volume at $z=15$ for our fiducial RSI model. If all the formed stars have a mass of $300 M_{\odot}$, the star-formation rate of $\sim 5 \times 10^{-4} M_{\odot} \mathrm{yr}^{-1} \mathrm{Mpc}^{-3}$ corresponds to the formation of 5 such stars every three million years within the volume. The calculation of Bromm et al. (2001) suggests that a total emission rate of $2.5 \times 10^{51}$ ionizing photons per second, a 50 times higher photon emission rate, can be maintained in this case, assuming such very massive stars are continuously formed. (Note that these massive stars are typically short-lived, with a main-sequence lifetime of about two million years.) We thus conclude that a 100 times higher photon production rate than assumed in our original RSI model serves as a conservative upper limit.

We examine the overall effect of such an additional photon supply by "boosting" the photon emission rate of the sources in the RSI massive-halo model. Sokasian et al. (2003a) found that boosting the photon emission rate at high redshifts progressively shifts the reionization epoch (see their Figure 13). Motivated by their result, we employ a simple prescription which increases the emission rate of individual sources according to

$$
\dot{N}_{\text {ph }, \text { eff. }}=\exp (0.53(z-6)) \times \dot{N}_{\text {ph }}
$$

where $\dot{N}_{\text {ph }}$ and $\dot{N}_{\text {ph,eff. }}$ are the original and the boosted photon emission rates, respectively. With this parametrization, the ionizing photon emission rate of the galaxies steeply increases toward higher redshift, modeling either a transition of the stellar IMF or a contribution from massive Population III stars or both. This rather extreme model requires effectively more than a 10 times higher photon emission rate at $z>10$, and more than 100 times higher at $z>15$. We carry out radiative transfer simulations for the RSI model with this luminosity "boost". Figure 12 shows the resulting $\tau_{e}(z)$. The reionization epoch is found to be shifted to earlier times, $z \sim 10$, than in 
the case considered in $\S 6.3$, and consequently $\tau_{e}$ is greatly increased compared with the fiducial RSI model. However, the total optical depth for this boost model reaches $\tau_{e} \sim 0.12$ at $z=0$, still lower than the $1-\sigma$ range of the WMAP result.

\section{SUMMARY}

Our numerical simulations of early structure formation show that primordial gas cloud formation in low-mass "mini-halos" is very inefficient at $z>15$ in the RSI model, making it unlikely that the "first stars" contribute significantly to reionization in this scenario. Using radiative transfer calculations, we also show that reionization by ordinary ("Population II") stellar sources in galaxies is completed late in the RSI model if we employ conventional models of star-formation. In order to be compatible with the epoch of the end stage of reionization inferred from high redshift quasar observations, the photon escape fraction from galaxies must be large, $f_{\text {esc }} \gtrsim 0.6$. We found that the resulting total Thomson optical depth in this case is $\tau_{e} \approx 0.055$, in apparent conflict with the recent WMAP measurements of CMB polarization.

In order for the total optical depth to be as large as $\tau_{e}>0.1$, reionization must be completed before $z \sim 10$ (Figure 7). Ciardi et al. (2003) and Wyithe \& Loeb (2003) suggest that employing an effectively top-heavy IMF may be necessary to cause early reionization. Cen (2003) argues that a large Thomson optical depth could be obtained if the "Population III era" is prolonged so that massive stars continuously form until quite low redshift. If a large volume of the IGM could be kept chemically pristine until low redshift $(z \sim 10)$ due to the very low global star-formation rate in the RSI model, the stellar IMF in galaxies may be top-heavy (Schneider et al. 2002; Mackey, Bromm \& Hernquist 2003). Unfortunately, little is presently known about star-formation in a primordial gas in high mass halos - the "first galaxies" - and clearly needs to be addressed using numerical simulations as well as theoretical modeling (Larson 1998; Omukai 2001; Oh \& Haiman 2002). We have explored the possibility of enhancing the photon emission rate by employing a simple prescription. The model parameters were chosen such that the photon emission rate is close to a plausible maximum bound. Our analysis has shown that, whereas the extreme boost model does predict an earlier reionization epoch $z_{\text {reion }} \sim 10$ and a larger total optical depth $\tau_{e} \sim 0.12$, the result is still marginally inconsistent with the WMAP data.

Recent numerical simulations by Bromm, Yoshida \& Hernquist (2003) show that early Population III supernovae quickly pollute the surrounding IGM with metals. If many massive population III stars are formed in "minihalos" and explode as hyper-energetic supernovae, the global metal-enrichment of the IGM is quickly achieved to such a degree that a transition of the stellar IMF is caused at a very early epoch (Yoshida, Bromm \& Hernquist 2003). Then, the large photon production rate at $z>6$ we hypothesized as coming from metal-free stars cannot be maintained for a sufficiently long time, and the reionization history in the RSI model will be just as shown in Figure 9] with a small total optical depth $\left(\tau_{e}<0.06\right)$ as shown in Figure 10.

In the end, we are left with a contradiction: the running of the primordial power spectrum favored by the WMAPext $+2 \mathrm{dF}+$ Lyman- $\alpha$ analysis and the high Thomson optical depth measured by the WMAP satellite appear to be inconsistent with one other. We have discussed various possibilities to resolve the conflict, and conclude that a rather radical solution or a fine-tuned combination, if any is possible, of the proposed resolutions are necessary. Models with a weaker running of the primordial power spectrum will alleviate the discrepancy slightly, but the overall results would not be changed. If the high Thomson optical depth is confirmed and the running of the primordial power spectrum turns out to be real, it may be necessary to invoke exotic radiation sources other than stellar populations or some ionization mechanisms. Such scenarios include decaying particles (Sciama 1982; Dodelson \& Jubas 1992; Hansen \& Haiman 2003) or formation of early mini-quasars (Eisenstein \& Loeb 1995; Sasaki \& Umemura 1996).

The prospects for observationally resolving this discrepancy in the near future appear bright. Data from planned CMB polarization experiments, by the continued operation of WMAP, and post-WMAP observatories such as Planck will pin down a precise value for $\tau_{e}$. Lyman$\alpha$ forest observations exploiting a large sample of SDSS quasars will place a tighter constraint on the matter power spectrum on large scales, as will the SDSS galaxy redshift survey. In the longer term, it may be possible to map out the evolution of reionization from redshifted $21 \mathrm{~cm}$ emission using instruments such as the Square Kilometer Array $^{\dagger}$ or the Low Frequency Array ${ }^{\ddagger}$ (e.g. Madau et al. 1997; Tozzi et al. 2000; Iliev et al. 2002; Ciardi \& Madau 2003). For example, Furlanetto et al. (2003) show that frequency fluctuations can be used to distinguish between models with a different number of reionization epochs. Analyses of these various high-precision data promise to provide a more complete picture of the matter density distribution in the early Universe over a wide range of scales and its relationship to the formation of stars and galaxies.

We thank James Bullock, Volker Bromm, and Ravi Sheth for helpful comments on the earlier draft. NY acknowledges support from the Japan Society of Promotion of Science Special Research Fellowship. This work was supported in part by NSF grants ACI 96-19019, AST 9802568, AST 99-00877, and AST 00-71019. The simulations were performed at the Center for Parallel Astrophysical Computing at the Harvard-Smithsonian Center for Astrophysics.

\footnotetext{
${ }^{\dagger}$ http://www.usska.org

$\ddagger_{\text {http://www.lofar.org }}$
} 


\section{REFERENCES}

Abel, T., Anninos, P., Norman, M. L., \& Zhang, Y. 1997, New Astronomy, 2, 181

Abel, T., Bryan, G. L., \& Norman, M. L. 2002. Science, 295, 93

Avelino, P.P. \& Liddle, A.R. 2003, astro-ph/0305357

Becker, R. H. et al. 2001, AJ, 122, 2850

Bromm, V., Kudritzki, R. P., \& Loeb, A. 2001, ApJ, 552, 464

Bromm, V., Coppi, P. S., \& Larson, R. B. 2002, ApJ, 564, 23

Bromm, V.. Yoshida, N., \& Hernquist, L. 2003, ApJL in press astro-ph/0305333

Bullock, J. S. \& Zentner, A. R. 2002 astro-ph/0207534

Cen, R., 2003, ApJ, 591, 12

Chiba, M., 2002, ApJ, 565, 17

Chiu, W. A., Fan, X. \& Ostriker, J. P. 2003, astro-ph/0304234

Ciardi, B., Ferrara, A. \& White, S. D. M., 2003, astro-ph/U3U2451

Ciardi, B. \& Madau, P., 2003, ApJ, submitted astro-ph/U3U3249

Cole, S., 1997, MNRAS, 286, 38

Covi, L. \& Lyth, D. H., 1999, PRD, 59, 63515

Croft, R. A. C., Weinberg, D. H., Katz, N. \& Hernquist, L., 1998, ApJ, 495, 44

Croft, R. A. C., Di Matteo, T. Davé, R., Hernquist, L., Katz, N., Fardal, M.A., Weinberg, D. H., 2001, ApJ, 557, 67

Croft, R. A. C., Weinberg, D. H., Bolte, M., Burles, S., Hernquist,

L., Katz, N., Kirkman, D. \& Tytler, D., 2002, ApJ, 581, 20

Dalal, N. \& Kochanek, C. S. 2002, ApJ, 572, 25

Dodelson, S, \& Jubas, J. M., 1992, PRD, 45, 1076

Eisenstein, D. J. \& Loeb, A. 1995, ApJ, 443, 11

Furlanetto, S.. Sokasian. A. \& Hernquist, L., 2003, MNRAS, submitted astro-ph/0305065

Galli, D. \& Palla, H. 1998, A\&A, 335, 403

Gnedin, N. Y. \& Hamilton, A. J. S.. 2002. MNRAS, 334, 107

Haiman, Z. \& Holder, G., 2003, astro-ph/0302403

Hannestad, S., Hansen, S. H., Villante, F. L. \& Hamilton, A. J. S., 2002, Astroparticle Physics, 17, 375

Hansen, S. H. \& Haiman, Z., 2003, astro-ph/0305126

Hernquist, L., 1993, ApJ, 404, 717

Hernquist, L. \& Springel, V., 2003, MNRAS, 341, 1253 astro-ph/0209183

Iliev, 1.1., Shapıro, P.R., Ferrara, A., Martel, H., 2002, ApJ, 572, L123

Jang-Condell, H. \& Hernquist, L., 2001, ApJ, 548, 68

Jenkins, A., Frenk, C. S., White, S. D. M., Colberg, J. M., Cole, S., Evrard, A. E., Couchman, H. M. P., Yoshida, N., 2001, MNRAS, 321,372

Kauffmann, G., Colberg, J. M., Diaferio, A. \& White, S. D. M., 1999, MNRAS, 303, 188

Kennicutt, R. C., 1998, ApJ. 498, 541

Kogut, A. et al., 2003, astro-ph/0302213

Kosowsky, A. \& Turner, M. S., 1995, PKD, 52, 1739

Larson, R. B., 1998, MNRAS, 569, 1998

Leitherer, C. et al., 1999, ApJS, 123, 3

Lyth, D. H. \& Riotto, A. A. 1999 Phys. Rep. 314, 1

Machacek, M., Bryan, G. L. \& Abel, T., 2001, ApJ, 548, 509

Mackey, J., Bromm, V. \& Hernquist, L. 2003, ApJ, 586, 1

Madau, P., Haardt, F. \& Rees, M. J., 1999, ApJ, 514, 648

Madau, P., Meiksin, A. \& Rees, M. J., 1997, ApJ, 475, 429
Matsuda, T., Sato, H. \& Takeda, H. 1969, Prog. Theor. Phys., 42, 219

McDonald, P. et al., 2000, ApJ, 543, 1

Moore, B., Ghigna, S., Governato, F., Lake, G., Quinn, T., Stadel, J. \& Tozzi, P., 1999 ApJL, 524, 19

Oh, S.-P., Nollett, K. M., Madau, P. \& Wasserburg, G. J., 2001, ApJ, 562, 1

Oh, S. P. \& Haiman, Z., 2002, ApJ, 569, 558

Omukai, K. \& Nishi, R. 1999, ApJ, 518, 64

Omukai, K. 2001, ApJ, 546, 635

Pearce et al. 1999 ApJ, 521, 99

Peiris, H. V., 2003, astro-ph/0302225

Reed, D. et al. 2003, astro-ph/0301270

Salpeter, E. E., 1955, ApJ, 121, 101

Sasaki, S. \& Umemura, M. 1996, ApJ, 462, 104

Schaerer, D., 2003, A\&A, 397, 527

Schneider, R., Ferrara, A., Natarayan, P. \& Omukai, K., 2002, ApJ, 571,30

Schwarzschild, M. \& Spitzer, L., 1953, Observatory, 73, 77

Seljak, U., McDonald, P. \& Makarov, A., 2003, MNRAS, 342, 79

Sheth, R. K. \& Tormen, G., 1999, MNRAS, 308, 119

Sheth, R. K. \& Tormen, G., 2002, MNRAS, 329, 61

Sokasian, A., Abel, T. \& Hernquist, L., 2001, NewA, 6, 359

Sokasian, A., Abel, T., Hernquist, L. \& Springel, V. 2003a, MNRAS in press astro-ph/0303098

Sokasian, A., Yoshıda, N., Abel, T., Hernquist, L. \& Springel, V. 2003b, MNRAS submitted [astro-ph/

Somerville. R.. Bullock, J. S. \& Livio, M, 2003, ApJ in press, astro-ph/0303481

Spergel, D. N. et al. 2003, astro-ph/0302209

Springel, V., White, S. D. M., Kauttmann, G. \& Tormen, G., 2001, MNRAS, 328, 726

Springel, V. \& Hernquist, L. 2002, MNRAS, 333, 649

Springel, V. \& Hernquist, L. 2003a, MNRAS, 339, 289

Springel, V. \& Hernquist, L. 2003b, MNRAS, 339, 312

Sugiyama, N., 1995, ApJ, 100, 281

Tegmark, M., Silk, J., Rees, M., Blanchard, A., Abel, T., \& Palla, F., 1997, ApJ, 474, 1

Thumlinson, J. \& Shull, J. M., 2000, ApJ, 528, L65

Tormen, G. \& Bertschinger, E., 1996, MNRAS, 472, 14

Tozzi, P., Madau, P., Meiksin, A. \& Rees, M., 2000, ApJ, 528, 597

Wyithe, J. S. \& Loeb, A., 2003, ApJL, 588, 69

Yoshida, N., Stoehr, F., Springel, V. \& White, S. D. M., 2002, MNRAS, 335, 762

Yoshida, N., Abel, T., Hernquist, L. \& Sugiyama, N., 2003a, ApJ, 592,645

Yoshida, N., Sokasian, A., Hernquist, L. \& Springel, V., 2003b, ApJL, 591, 1

Yoshida. N.. Sugivama, N. \& Hernquist, L., 2003, MNRAS, in press astro-ph/0305210

Yoshida, N., Bromm, V., \& Hernquist, L. 2003, in preparation

Yoshii, Y. \& Saio, H., 1986, ApJ, 301, 587

Zentner, A. R. \& Bullock, J. S., 2002, PRD, 66, 043003

Zentner, A. R. \& Bullock, J. S., 2003, ApJ in press astro-ph/0304292 